
$\$$ Research Square
Preprints are preliminary reports that have not undergone peer review.
They should not be considered conclusive, used to inform clinical practice, or referenced by the media as validated information.

\title{
Bioinformatics analysis of differentially expressed genes and their potentially interacting miRNAs in oral lichen planus
}

Churen Zhang ( $18311321821 @ 163 . c o m)$

The First Affiliated Hospital of Xiamen University https://orcid.org/0000-0001-9070-7138

Ruoran Sun

Beijing Daxiang Biotech Co,.Ltd

\section{Research Article}

Keywords: Computational Biology, Bioinformatics, Oral lichen planus, miRNA

Posted Date: October 13th, 2021

DOI: https://doi.org/10.21203/rs.3.rs-952216/v2

License: () (7) This work is licensed under a Creative Commons Attribution 4.0 International License. Read Full License 


\section{Abstract}

Background. Among the diseases of oral mucosa, oral lichen planus (OLP) is characterized by chronic autoimmune/autoinflammation. However, the etiology and pathogenesis of OLP were still limited. The aim of this research was to identify the differentially expressed genes and their potentially interacted miRNAs in OLP to provide a possible explanation of the pathogenesis of OLP and therapeutic biomarkers.

Methods. The OLP microarray dataset (GSE52130) was download from the Gene Expression Omnibus (GEO) database. R software was used to identify differentially expressed genes between the OLP samples and normal oral mucosa. Functional enrichment analysis of DEGs, including Gene Ontology (GO) and Kyoto Encyclopedia of Genes and Genomes (KEGG) pathway, were conducted. Protein-protein interaction (PPI) network analysis was performed in the STRING database. CytoHubba in the Cytoscape software was applied to determining the top 10 hub genes, whose relative miRNA was identified through RNA Interactome Database.

Results. Overall, 627 DEGs was identified in OLP samples, including 351 highly expressed genes and 276 lowly expressed genes. GO analysis indicated that the epidermal differentiation was mostly enriched. For the KEGG pathway, the DEGs in OLP samples were mostly involved in Staphylococcus aureus infection, Estrogen signaling pathway, Serotonergic synapse and Histidine metabolism. Top 10 hub genes including LOR, LCE3D, LCE3E, LCE1B, LCE2B, SPRR2E, SPRR2G, LCE2A, RPTN and CDSN were identified from the PPI network. The miRNA (hsa-miR-98-5p) was regarded as the mostly possible miRNA involved in OLP.

\section{Introduction}

Among the diseases of oral mucosa, oral lichen planus (OLP) was characterized by chronic autoimmune/autoinflammation.[1] OLP markedly affected middleaged adults, women more than men.[2] The typical lesions were characterized by small papules the size of a needle connected into white or off-white fine streaks, which were intertwined into a network, dendritic or ring shape.[3] Most of the lesions appeared as asymptomatic, congestive erosions and ulcers, and cheek was the most common site of OLP occurrence.[4] Patients with OLP were usually asymptomatic. The lesions of OLP might feel rough, burning and dried, which affected the function of eating and speech of the patient.[5] It had been reported that the incidence of OLP in the general population was about $2 \%$.[6] A recent meta-analysis showed that the incidence of OLP differed in different regions, with a slightly higher incidence in European population (1.43\%) and a slightly lower incidence in Asian population (0.49\%).[7] OLP lied in the potential to develop into malignancy and was classified as a premalignant condition by the World Health Organization.[3] However, the etiology and pathogenesis of OLP were still limited, and they were believed to be related to various factors, such as immune factors, mental factors, and infectious factors.[8]

Identification of biomarkers of OLP contributed to a better understanding of its underlined molecular mechanisms, and provide a basis for clinical personalized and precise treatment.[9]

Abnormal expressions of disease-related genes were believed to contribute to OLP pathogenesis.[10,11] However, there was actually no gold standard therapeutic and prognostic biomarker for OLP. Therefore, it was necessary to explore new biomarkers for OLP.

In recent years, development of high-throughput technologies had provided a new perspective for studying the pathogenesis of diseases.[12] It had been applied to explore the differentially expressed genes in disease progression and determine disease-related biomarkers.[13] As an emerging research method, clinical bioinformatics could identify abnormally expressed genes in diseased tissues by analyzing the gene profiles obtained from high-throughput microarray and sequencing technology, which was considered to be one of the promising and key methods to help achieve early diagnosis and effective treatment.[14]

This study used bioinformatics methods to perform differential gene analysis and functional cluster analysis based on OLP transcription microarray from public databases. Moreover, protein-protein interaction networks and related miRNA networks were established. The aim of this research was to identify the differentially expressed genes and their potentially interacting miRNAs in OLP to provide a possible explanation of the pathogenesis of OLP and potential biomarkers.

\section{Materials And Methods}

\section{Source of data}

The OLP microarray dataset (GSE52130) was download from the GEO database (https://www.ncbi.nlm.nih.gov/geo/query/acc.cgi?acc=GSE52130) through the GEO query package.[15] The dataset was based on GPL10558 Illumina HumanHT-12 V4.0 expression beadchip. As described in the original research,[10] 7 patients, who were clinically and histologically diagnosed as OLP, and 8 controls with healthy mucosa were included for biopsy collection. The age of all participants ranged from 45-69 years old, without significant difference between two groups. Finally, 14 samples including 7 OLP samples and 7 normal oral epithelium samples were incorporated in this research.

\section{Preprocessing of data}

Bioconductor package (3.13 version) of R software (4.1.0) was used to preprocess the data. The probes corresponding to multiple molecules were removed. When various probes corresponded to the same molecule, only the probe with the largest signal value was reserved. Then the box diagram was conducted 
through ggplot2 (3.3.3 version) to estimate the standardization of the samples, and the clustering analysis between the OLP group and the normal group was conducted by umap package (0.2.7.0 version).

\section{Differentially expressed genes analysis}

Linear models were fitted by limma package of R software (4.1.0) to further determine the differential expressed genes (DEGs) between the OLP group and normal group.[16] Benjamini Hochberg was used for multiple comparisons. DEGs were defined as genes with adjust $P$-value $<0.05$ and $\mid$ log2(FC) $\mid>1.0$.

\section{Functional enrichment analysis of DEGs}

Gene Ontology (GO) (http://www.geneontology.org/) and Kyoto Encyclopedia of Genes and Genomes (KEGG) pathways (https://www.genome.jp/kegg/) were analyzed for the DEGs. P-value $<0.05$ was indispensable for the GO/KEGG terms, which were determined as significantly enriched.

\section{Protein-protein interaction (PPI) network analysis}

STRING (https://string-db.org/), a database of known and predicted PPI, was applied to the identification of protein interactions. Cytoscape software (3.8.2 version) was used to visualize the PPI. In addition, CytoHubba, a plug-in of Cytoscape, was used to identify the top 10 hub genes through Maximal Clique Centrality algorithm.[17]

\section{MiRNA network of top 10 hub genes}

RNA Interactome Database (http://www.rnainter.org/) was screened for the relevant miRNAs of top 10 hub genes.[18] The interaction score should not be lower than 0.2. The top 30 relevant miRNAs were included. The miRNAs, targeting at least 3 hub genes, were identified.

\section{Results}

\section{DEGs of OLP}

The median of each gene profile was basically on a horizontal line, and it indicated that the degree of normalization between samples was good. (Figure 1A) Box plot elements were showed in Table 1. The samples of each group were remarkably separated in the UMAP figure, showing that the distribution of data in both groups was obviously different. (Figure 1B) According to the definition of DEGs, a total of 627 DEGs was identified in OLP samples, including 351 highly expressed genes and 276 lowly expressed genes. (Figure 1C) The expression of top 20 highly expressed genes and top 20 lowly expressed genes was visualized in the heatmap. (Figure 1D)

\section{Functional enrichment of DEGs}

GO enrichment included the functional information of biological process (BP), molecular function (MF) and cellular component (CC), in which the DEGs were involved. For the BP, the DEGs in OLP samples were mostly associated with epidermis development, skin development, epidermal cell differentiation and keratinocyte differentiation. (Figure 2A) For the MF, the DEGs in OLP samples were mostly involved in enzyme inhibitor activity, peptidase regulator activity, endopeptidase regulator activity, peptidase inhibitor activity and endopeptidase inhibitor activity. (Figure 2B) For the CC, the DEGs in OLP samples were mostly related to cornified envelope, intermediate filament cytoskeleton and intermediate filament. (Figure 2C) For the KEGG pathway, the DEGs in OLP samples were mostly involved in Staphylococcus aureus infection, Estrogen signaling pathway, Serotonergic synapse and Histidine metabolism. (Figure 2D)

\section{PPI network construction}

STRING database was used for PPI analysis of DEGs, and further Cytoscape software was applied to analyze and visualize the results. (Figure 3A) Moreover, top 10 genes were identified as the possible hub genes by the CytoHubba, including LOR, LCE3D, LCE3E, LCE1B, LCE2B, SPRR2E, SPRR2G, LCE2A, RPTN and CDSN. (Figure 3B) The top 10 hub genes were all upregulated in OLP samples.

\section{MiRNA network of top 10 hub genes}

RNA Interactome Database was used for the establishment of the mRNA-miRNA interaction network. (Figure 4) For example, the miRNA (hsa-miR-98-5p) was found to interact with 5 hub genes (LOR, SPRR2G, LCE2B, CDSN and LCE2A). The miRNA (hsa-let-7e-5p, hsa-let-7f-5p, hsa-let-7d-5p, hsa-miR-135a-5p, hsa-miR202-3p, hsa-miR-326, hsa-miR-708-5p, hsa-miR-330-5p, hsa-miR-28-5p) was found to interact with 3 different hub genes. (Figure 5)

Page $3 / 10$ 


\section{Discussion}

OLP was a common oral mucosal disease. However, the pathogenesis of OLP was limited, and it was believed to be associated with multiple factors. Notably, There are a paucity of effective therapies for the treatment and prevention of OLP.[19] The molecular mechanisms of OLP remained to be investigated for further identifying the potentially effective therapeutic targets. Bioinformatics was one of the promising methods to find the abnormally expressed genes for further identification of therapeutic targets. In this research, we used a gene series of OLP from GEO database to find 627 DEGs in OLP samples, including 351 highly expressed genes and 276 lowly expressed genes. Functional enrichment analysis found the DEGs enrich in epidermis development and enzyme inhibitor activity. The function of hub genes was almost related to the cornified cell envelope formation and epidermal barrier integrity.[20-22]

Loricrin cornified envelope precursor protein (LOR) encoded loricrin, which was one of major proteins in the envelope of terminally differentiated epidermal keratinocytes.[23] Mutations in LOR were related to Vohwinkel syndrome, which was defined as palmoplantar keratoderma.[24] LOR had been found to be associated with cornified cell envelope formation, which resulted in keratinization in the OLP lesions. [21] It was consistent with our results. However, another study had indicated that loricrin was not detected in OLP samples through immunohistochemical technique.[25] Whether LOR could be regarded as a biomarker for OLP needed more researches.

Late cornified envelope (LCE) family included multiple genes, which encoded stratum-corneum proteins.[26] LCE genes had been found to be important in skin and upregulate under the UV light.[21] They were the precursors of the cornified envelope of the stratum corneum.[27] Therefore, they participated in the keratinization pathway, which was involved in the pathology of OLP.[28] Moreover, LCE proteins were identified as antimicrobial activator to be closely connected with the innate host defense, indicating their potential function in the pathogenesis of OLP.[29] The LCE genes not only correlate to keratinization, but also involved the immune response, both of which were the possibly major etiology of OLP. Nevertheless, up to now, few studies focused on the function of LCE in the development of OLP.

Small proline rich protein (SPRR) genes encoded small proline rich proteins, which participated the epidermal differentiation complex to further constitutes the cornified cell envelope.[30] In addition, repetin (RPTN) gene, belonging to S100 protein family, was also involved in the cornified cell envelope formation. The LOR, LCE, SPRR and RPTN were parts of human epidermal differentiation complex (EDC).[21] The GO analysis in this study enriched the epidermal differentiation. It was indicated that upregulated or activated EDC might play a role in the pathogenesis of OLP.

Another bioinformatic study had indicated that high expression of filaggrin (FLG) gene was related to hyperkeratosis, and arachidonate 12-lipoxygenase, 12R type (ALOX12B) gene could aggravate wound in the oral mucosa.[31] Moreover, FLG and ALOX12B were found to be involved in OLP.[32] FRAS1 gene was indicated to be related to separation between lamina propria and epithelium in a bioinformatic research.[32] The identified hub genes of OLP in previous studies were different from that in our study.[9,10] Our research found new potential biomarkers. More basic and clinical researches were urged to prove the function of these genes in the pathogenesis of OLP.

MiRNA was a kind of endogenous non-coding RNA, extensively participating in gene post-transcriptional regulation activities.[33] MiRNA could inhibit target gene expression at protein translation level and induce the degradation of target mRNA.[34] MiR-164a and miR-21 were found to be upregulated over 2-fold in the OLP samples.[35] MiR-155 targeted suppressor of cytokine signaling 1 (SOCS1) to relieve the inhibition of phosphorylated janus kinase (JAK), then signal transducer and activator of transcription (STAT) was activated to M1 polarization and Treg differentiation, which promoted the development of OLP.[36] The miRNAs identified in the present study remained to be further investigated.

Our study provided a new direction for mechanism research of OLP. The role of EDC relevant genes and corresponding miRNAs in OLP remained to be studied. Nonetheless, there were some limitations in the present research. (1) The function of hub genes and miRNAs in OLP was less studied; (2) The interaction between hub genes and miRNAs was not investigated. However, the results of this research were still meaningful, as potential biomarkers for diagnosis and treatment of OLP were provided.

\section{Conclusions}

Compared to the normal oral mucosa, genes, including LOR, LCE3D, LCE3E, LCE1B, LCE2B, SPRR2E, SPRR2G, LCE2A, RPTN and CDSN, were significantly highly expressed in OLP samples. These genes were parts of the EDC, referring to the potential role of EDC in the pathogenesis of OLP. Possibly functional miRNAs (hsa-miR-98-5p, hsa-let-7e-5p, hsa-let-7f-5p, etc) were also identified. Our results might help to identify the biomarkers of OLP. However, more basic and clinical experiments were needed to validate the role of above molecules in OLP.

\section{Declarations}

\section{Acknowledgement}

The authors thanks for the work of editors of this article.

\section{Author contributions}

Zhang Churen: Conceptualization, Methodology, Data curation, Software, Writing- Original draft preparation. Sun Ruoran: Visualization, Investigation, Supervision, Validation. Writing- Reviewing and Editing, 


\section{Conflicts of interest}

No conflict of interest was involved in the present research

\section{Ethical approval}

Ethical approval is not required for this type of study

\section{References}

[1] S. Hasan, S. Ahmed, R. Kiran, R. Panigrahi, J.M. Thachil, S. Saeed, Oral lichen planus and associated comorbidities: An approach to holistic health, J Family Med Prim Care 8 (2019) 3504-3517. 10.4103/jfmpc.jfmpc_749_19.

[2] M.Z. Mutafchieva, M.N. Draganova-Filipova, P.I. Zagorchev, G.T. Tomov, Oral Lichen Planus - Known and Unknown: a Review, Folia Med (Plovdiv) 60 (2018) 528-535. 10.2478/folmed-2018-0017.

[3] M.A. Gonzalez-Moles, P. Ramos-Garcia, S. Warnakulasuriya, An appraisal of highest quality studies reporting malignant transformation of oral lichen planus based on a systematic review, Oral Dis (2020). 10.1111/odi.13741.

[4] L.M. DeAngelis, N. Cirillo, M.J. McCullough, The immunopathogenesis of oral lichen planus-ls there a role for mucosal associated invariant T cells?, J Oral Pathol Med 48 (2019) 552-559. 10.1111/jop.12898.

[5] M. Carrozzo, S. Porter, V. Mercadante, S. Fedele, Oral lichen planus: A disease or a spectrum of tissue reactions? Types, causes, diagnostic algorhythms, prognosis, management strategies, Periodontol 200080 (2019) 105-125. 10.1111/prd.12260.

[6] Z.Y. Shen, W. Liu, L.K. Zhu, J.Q. Feng, G.Y. Tang, Z.T. Zhou, A retrospective clinicopathological study on oral lichen planus and malignant transformation: analysis of 518 cases, Med Oral Patol Oral Cir Bucal 17 (2012) e943-947. 10.4317/medoral.17778.

[7] M.A. Gonzalez-Moles, S. Warnakulasuriya, I. Gonzalez-Ruiz, L. Gonzalez-Ruiz, A. Ayen, D. Lenouvel, I. Ruiz-Avila, P. Ramos-Garcia, Worldwide prevalence of oral lichen planus: A systematic review and meta-analysis, Oral Dis 27 (2021) 813-828. 10.1111/odi.13323.

[8] K. Baek, Y. Choi, The microbiology of oral lichen planus: Is microbial infection the cause of oral lichen planus?, Mol Oral Microbiol 33 (2018) $22-28$. 10.1111/omi.12197.

[9] E.F. Zhong, A. Chang, A. Stucky, X. Chen, T. Mundluru, M. Khalifeh, P.P. Sedghizadeh, Genomic Analysis of Oral Lichen Planus and Related Oral Microbiome Pathogens, Pathogens 9 (2020). 10.3390/pathogens9110952.

[10] K. Danielsson, P.J. Coates, M. Ebrahimi, E. Nylander, Y.B. Wahlin, K. Nylander, Genes involved in epithelial differentiation and development are differentially expressed in oral and genital lichen planus epithelium compared to normal epithelium, Acta Derm Venereol 94 (2014) 526-530. 10.2340/00015555-1803.

[11] F. Agha-Hosseini, H. Barati, M.S. Moosavi, Aquaporin3 (AQP3) expression in oral epithelium in oral lichen planus, Exp Mol Pathol 115 (2020) 104441. 10.1016/j.yexmp.2020.104441.

[12] S. Das, C.J. McClain, S.N. Rai, Fifteen Years of Gene Set Analysis for High-Throughput Genomic Data: A Review of Statistical Approaches and Future Challenges, Entropy (Basel) 22 (2020). 10.3390/e22040427.

[13] Y. Rahmatallah, F. Emmert-Streib, G. Glazko, Gene set analysis approaches for RNA-seq data: performance evaluation and application guideline, Brief Bioinform 17 (2016) 393-407. 10.1093/bib/bbv069.

[14] K. Scherrer, Primary transcripts: From the discovery of RNA processing to current concepts of gene expression - Review, Exp Cell Res 373 (2018) 1-33. 10.1016/j.yexcr.2018.09.011.

[15] S. Davis, P.S. Meltzer, GEOquery: a bridge between the Gene Expression Omnibus (GEO) and BioConductor, Bioinformatics 23 (2007) $1846-1847$. 10.1093/bioinformatics/btm254

[16] I. Diboun, L. Wernisch, C.A. Orengo, M. Koltzenburg, Microarray analysis after RNA amplification can detect pronounced differences in gene expression using limma, BMC Genomics 7 (2006) 252. 10.1186/1471-2164-7-252.

[17] C.H. Chin, S.H. Chen, H.H. Wu, C.W. Ho, M.T. Ko, C.Y. Lin, cytoHubba: identifying hub objects and sub-networks from complex interactome, BMC Syst Biol 8 Suppl 4 (2014) S11. 10.1186/1752-0509-8-S4-S11.

[18] Y. Lin, T. Liu, T. Cui, Z. Wang, Y. Zhang, P. Tan, Y. Huang, J. Yu, D. Wang, RNAlnter in 2020: RNA interactome repository with increased coverage and annotation, Nucleic Acids Res 48 (2020) D189-D197. 10.1093/nar/gkz804.

[19] E.L. da Silva, T.B. de Lima, P.V. Rados, F. Visioli, Efficacy of topical non-steroidal immunomodulators in the treatment of oral lichen planus: a systematic review and meta-analysis, Clin Oral Investig 25 (2021) 5149-5169. 10.1007/s00784-021-04072-7.

Page 5/10 
[20] V. Oji, K.M. Eckl, K. Aufenvenne, M. Natebus, T. Tarinski, K. Ackermann, N. Seller, D. Metze, G. Nurnberg, R. Folster-Holst, M. Schafer-Korting, I. Hausser, H. Traupe, H.C. Hennies, Loss of corneodesmosin leads to severe skin barrier defect, pruritus, and atopy: unraveling the peeling skin disease, Am J Hum Genet 87 (2010) 274-281. 10.1016/j.ajhg.2010.07.005.

[21] B. Jackson, C.M. Tilli, M.J. Hardman, A.A. Avilion, M.C. MacLeod, G.S. Ashcroft, C. Byrne, Late cornified envelope family in differentiating epitheliaresponse to calcium and ultraviolet irradiation, J Invest Dermatol 124 (2005) 1062-1070. 10.1111/j.0022-202X.2005.23699.X.

[22] M. Trzeciak, M. Sakowicz-Burkiewicz, M. Wesserling, J. Glen, D. Dobaczewska, T. Bandurski, R. Nowicki, T. Pawelczyk, Altered Expression of Genes Encoding Cornulin and Repetin in Atopic Dermatitis, Int Arch Allergy Immunol 172 (2017) 11-19. 10.1159/000453452.

[23] Y. Ishitsuka, D.R. Roop, Loricrin: Past, Present, and Future, Int J Mol Sci 21 (2020). 10.3390/ijms21072271.

[24] G. Rovere, L. Stramazzo, A. Cioffi, N. Galvano, D. Pavan, G. Restuccia, A. D'Arienzo, R. Capanna, G. Maccauro, M. D'Arienzo, L. Camarda, What's the resolutive surgery for pseudo-ainhum in Vohwinkel syndrome? A case report and review of the literature, Orthop Rev (Pavia) 12 (2020) 8868.

10.4081/or.2020.8868.

[25] K. Shimada, T. Ochiai, H. Hasegawa, Ectopic transglutaminase 1 and 3 expression accelerating keratinization in oral lichen planus, J Int Med Res 46 (2018) 4722-4730. 10.1177/0300060518798261.

[26] H. Niehues, I.M. van Vlijmen-Willems, J.G. Bergboer, F.F. Kersten, M. Narita, W.J. Hendriks, E.H. van den Bogaard, P.L. Zeeuwen, J. Schalkwijk, Late cornified envelope (LCE) proteins: distinct expression patterns of LCE2 and LCE3 members suggest nonredundant roles in human epidermis and other epithelia, $\mathrm{Br} \mathrm{J}$ Dermatol 174 (2016) 795-802. 10.1111/bjd.14284.

[27] L. Bao, J. Li, B.E. Perez White, P.M. Patel, K.T. Amber, Inhibition of dipeptidyl-peptidase 4 induces upregulation of the late cornified envelope cluster in keratinocytes, Arch Dermatol Res (2021). 10.1007/s00403-021-02249-4.

[28] N.K. Archer, M.N. Dilolli, L.S. Miller, Pushing the Envelope in Psoriasis: Late Cornified Envelope Proteins Possess Antimicrobial Activity, J Invest Dermatol 137 (2017) 2257-2259. 10.1016/j.jid.2017.08.026.

[29] H. Niehues, L.C. Tsoi, D.A. van der Krieken, P.A.M. Jansen, M.A.W. Oortveld, D. Rodijk-Olthuis, I. van Vlijmen, W. Hendriks, R.W. Helder, J.A. Bouwstra, E.H. van den Bogaard, P.E. Stuart, R.P. Nair, J.T. Elder, P. Zeeuwen, J. Schalkwijk, Psoriasis-Associated Late Cornified Envelope (LCE) Proteins Have Antibacterial Activity, J Invest Dermatol 137 (2017) 2380-2388. 10.1016/j.jid.2017.06.003.

[30] F. Carregaro, A.C. Stefanini, T. Henrique, E.H. Tajara, Study of small proline-rich proteins (SPRRs) in health and disease: a review of the literature, Arch Dermatol Res 305 (2013) 857-866. 10.1007/s00403-013-1415-9.

[31] R. Iglesias-Bartolome, A. Uchiyama, A.A. Molinolo, L. Abusleme, S.R. Brooks, J.L. Callejas-Valera, D. Edwards, C. Doci, M.L. Asselin-Labat, M.W. Onaitis, N.M. Moutsopoulos, J.S. Gutkind, M.I. Morasso, Transcriptional signature primes human oral mucosa for rapid wound healing, Sci Transl Med 10 (2018). 10.1126/scitranslmed.aap8798.

[32] P.T. Vo, S.S. Choi, H.R. Park, A. Lee, S.H. Jeong, Y. Choi, Gene signatures associated with barrier dysfunction and infection in oral lichen planus identified by analysis of transcriptomic data, PLoS One 16 (2021) e0257356. 10.1371/journal.pone.0257356.

[33] S. Moazzendizaji, A. Sevbitov, F. Ezzatifar, H.R. Jalili, M. Aalii, M. Hemmatzadeh, S. Aslani, J.G. Navashenaq, R. Safari, R. Hosseinzadeh, M.R. Rahmany, H. Mohammadi, microRNAs: small molecules with a large impact on colorectal cancer, Biotechnol Appl Biochem (2021). 10.1002/bab.2255.

[34] K. Johansson, P.G. Woodruff, K.M. Ansel, Regulation of airway immunity by epithelial miRNAs, Immunol Rev (2021). 10.1111/imr.13028.

[35] V. Gassling, J. Hampe, Y. Acil, J.H. Braesen, J. Wiltfang, R. Hasler, Disease-associated miRNA-mRNA networks in oral lichen planus, PLoS One 8 (2013) e63015. 10.1371/journal.pone.0063015.

[36] Y. Tao, R. Ai, Y. Hao, L. Jiang, H. Dan, N. Ji, X. Zeng, Y. Zhou, Q. Chen, Role of miR-155 in immune regulation and its relevance in oral lichen planus, Exp Ther Med 17 (2019) 575-586. 10.3892/etm.2018.7019.

\section{Tables}

\begin{tabular}{|c|c|c|c|c|c|c|c|c|c|}
\hline & GSM1260095 & GSM1260096 & GSM1260097 & GSM1260098 & GSM1260099 & GSM1260100 & GSM1260101 & GSM1260102 & $\overline{\text { GSM1 }}$ \\
\hline median & 4.600035077 & 4.599655031 & 4.599792752 & 4.599966619 & 4.600180043 & 4.60005691 & 4.599777671 & 4.599759833 & 4.599 \\
\hline maxima & 14.27193272 & 14.27193272 & 14.27193272 & 14.27193272 & 14.27193272 & 14.27193272 & 14.27193272 & 14.27193272 & 14.27 \\
\hline minima & 4.246908353 & 4.246908353 & 4.246908353 & 4.246908353 & 4.246908353 & 4.246908353 & 4.246908353 & 4.246908353 & 4.246 \\
\hline $\begin{array}{l}25 \% \\
\text { percentiles }\end{array}$ & 4.446445929 & 4.446074678 & 4.446099312 & 4.446030751 & 4.446455458 & 4.446392748 & 4.446455458 & 4.446239347 & 4.446 \\
\hline $\begin{array}{l}75 \% \\
\text { percentiles }\end{array}$ & 5.701773289 & 5.701186624 & 5.701248498 & 5.700283824 & 5.701248498 & 5.701248498 & 5.701248498 & 5.701515536 & 5.701 \\
\hline
\end{tabular}

Table 1 Box plot elements were listed 
Figures
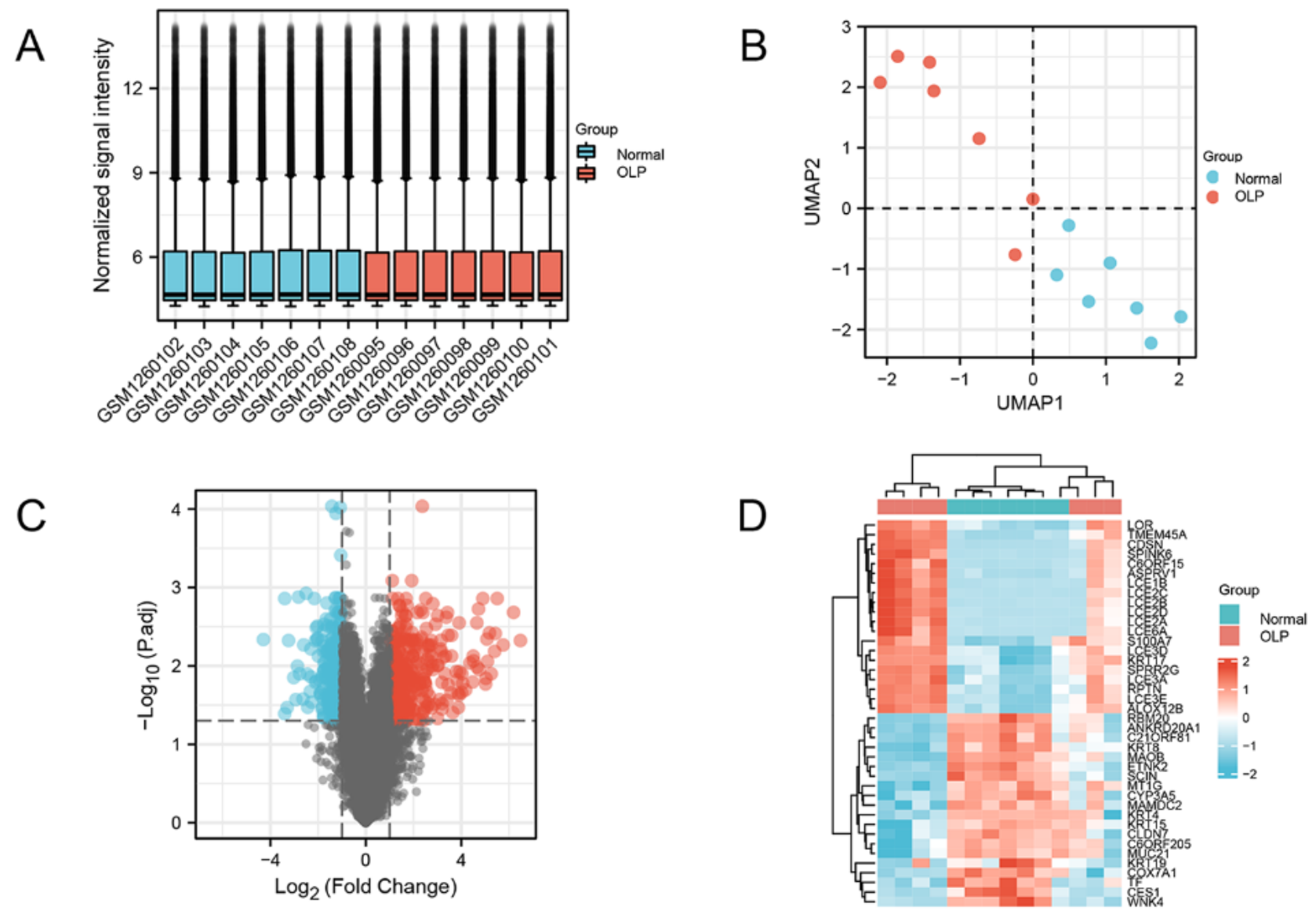

Figure 1

Data distribution and differentially expressed genes analysis. (A) mRNA level of the included microarray dataset; (B) UMAP plot showing the dataset differences between the normal oral mucosa group $(n=7)$ and OLP group $(n=7)$; $(C)$ Volcano plot showing the differentially expressed genes between the normal oral mucosa group and OLP group (627 DEGs). Benjamini Hochberg was used for multiple comparisons. Adjust $P$ value $<0.05$. Limma package of $R$ software was used to determine the DEGs; (D) Heatmap showing the expression of top 20 genes with high and low expression. 
A

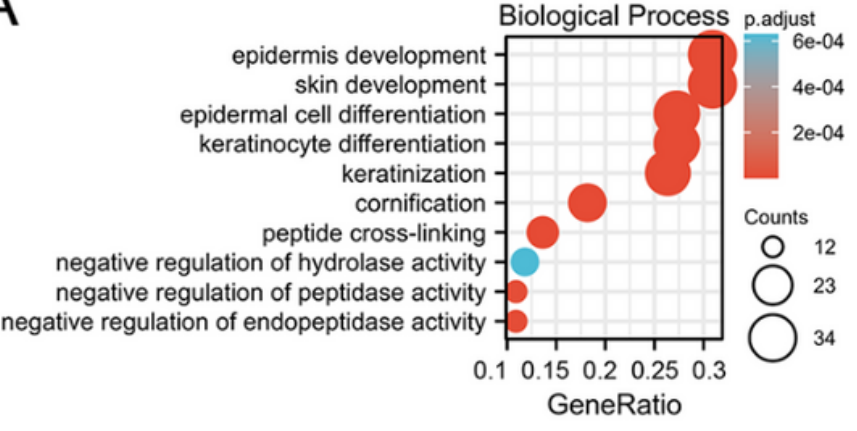

C

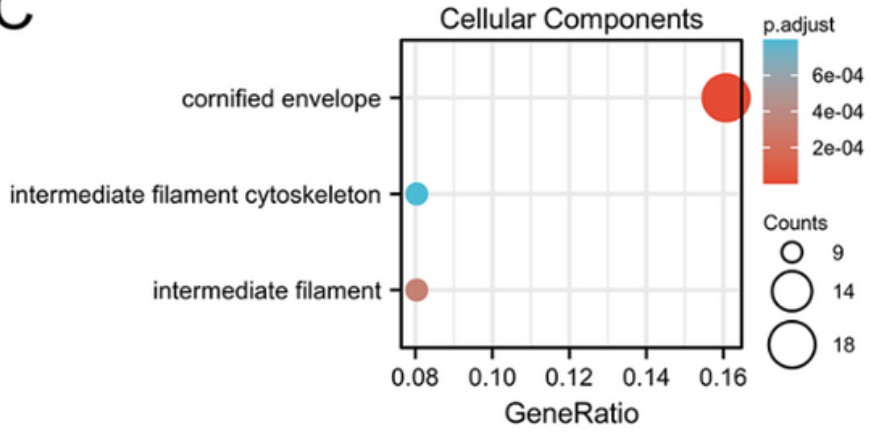

B

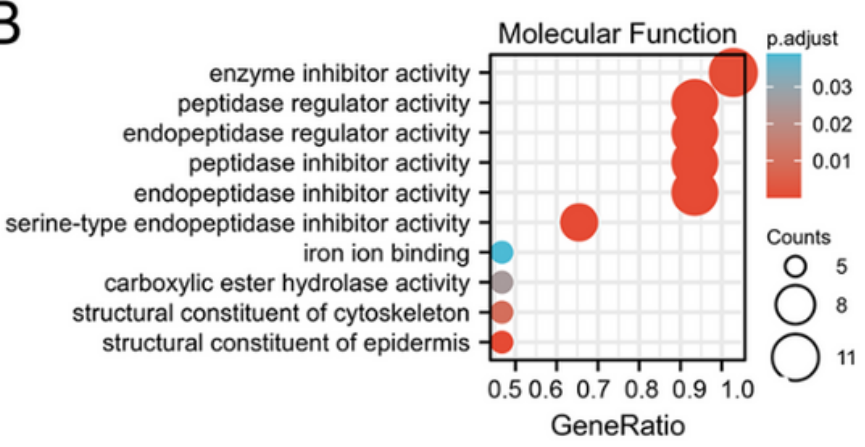

D

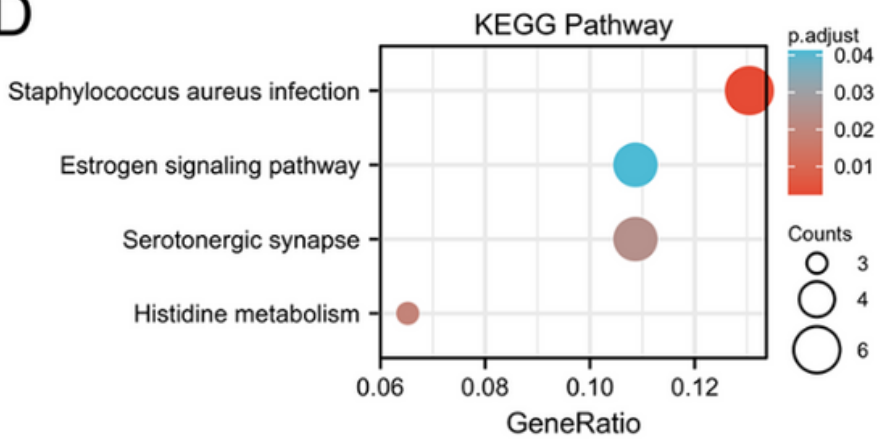

Figure 2

Significantly enriched GO terms and KEGG pathways of the 627 DEGs (A) Top 10 enriched biological process; (B) Top 10 enriched molecular function; (C) Top 10 enriched cellular component; (D) Top 10 enriched KEGG pathways. ClusterProfiler package of R software was performed for the enrichment analysis. Benjamini Hochberg was used for multiple comparisons. Adjust $P$ value $<0.05$.

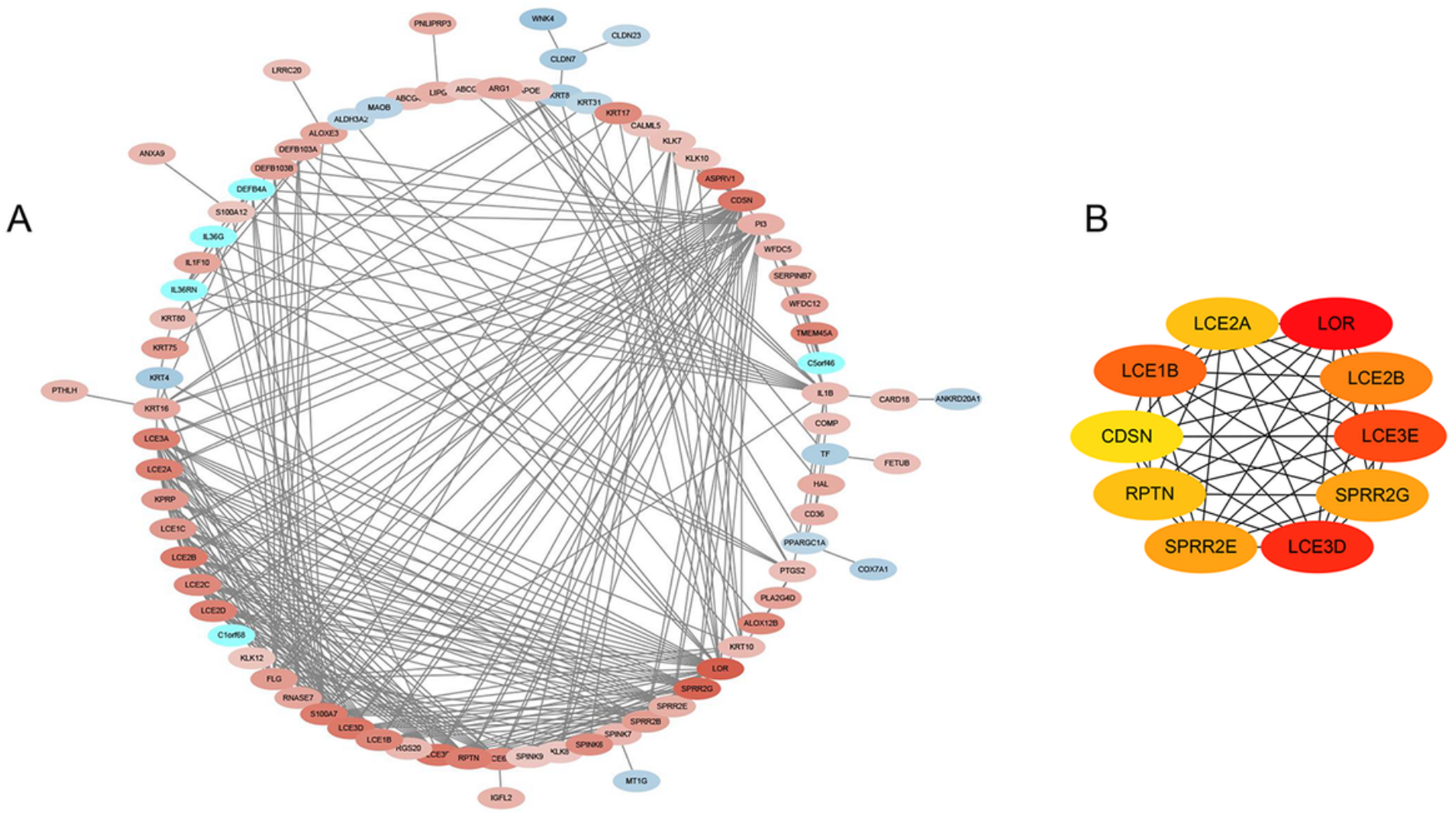

Figure 3 
The network of protein-protein interaction and top 10 hub genes. (A) Protein-protein interaction of the differentially expressed genes; (B) The top 10 hub genes identified through CytoHubba.

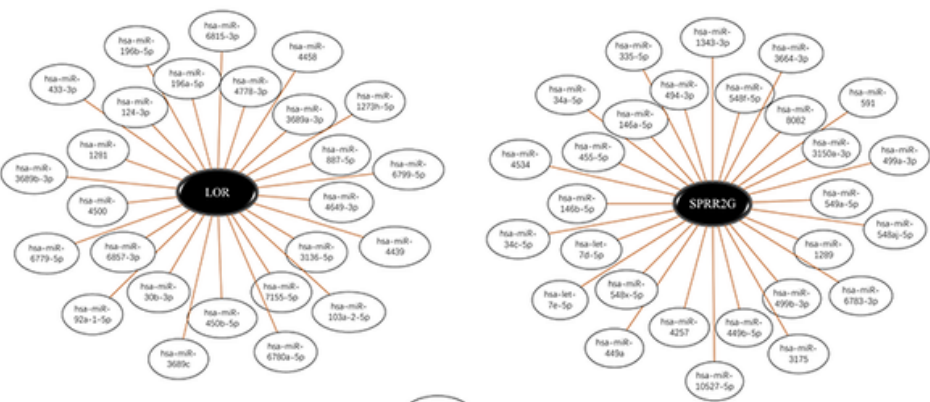

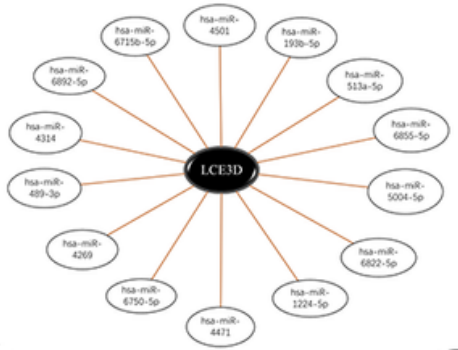

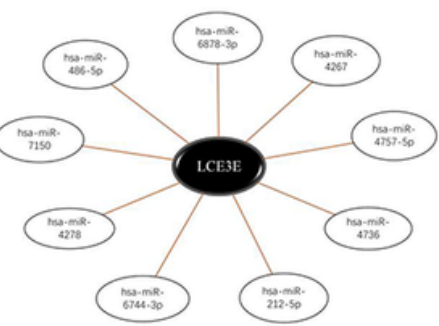

$$
\begin{aligned}
& \text { inisicis } \\
& \text { (ixic) } \\
& \text { (ixis) } \\
& \text { (ind) } \\
& \text { (int) }
\end{aligned}
$$
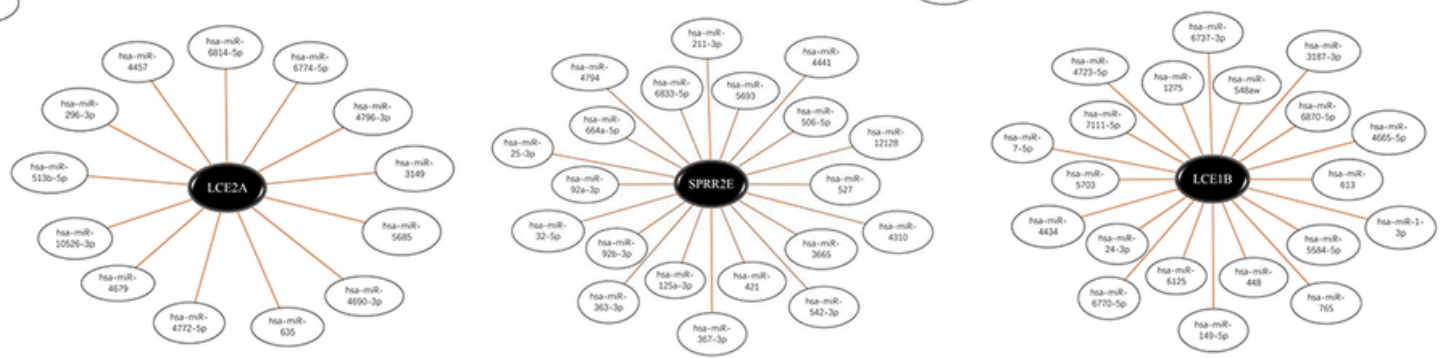

Figure 4

MiRNA network of top 10 hub genes.

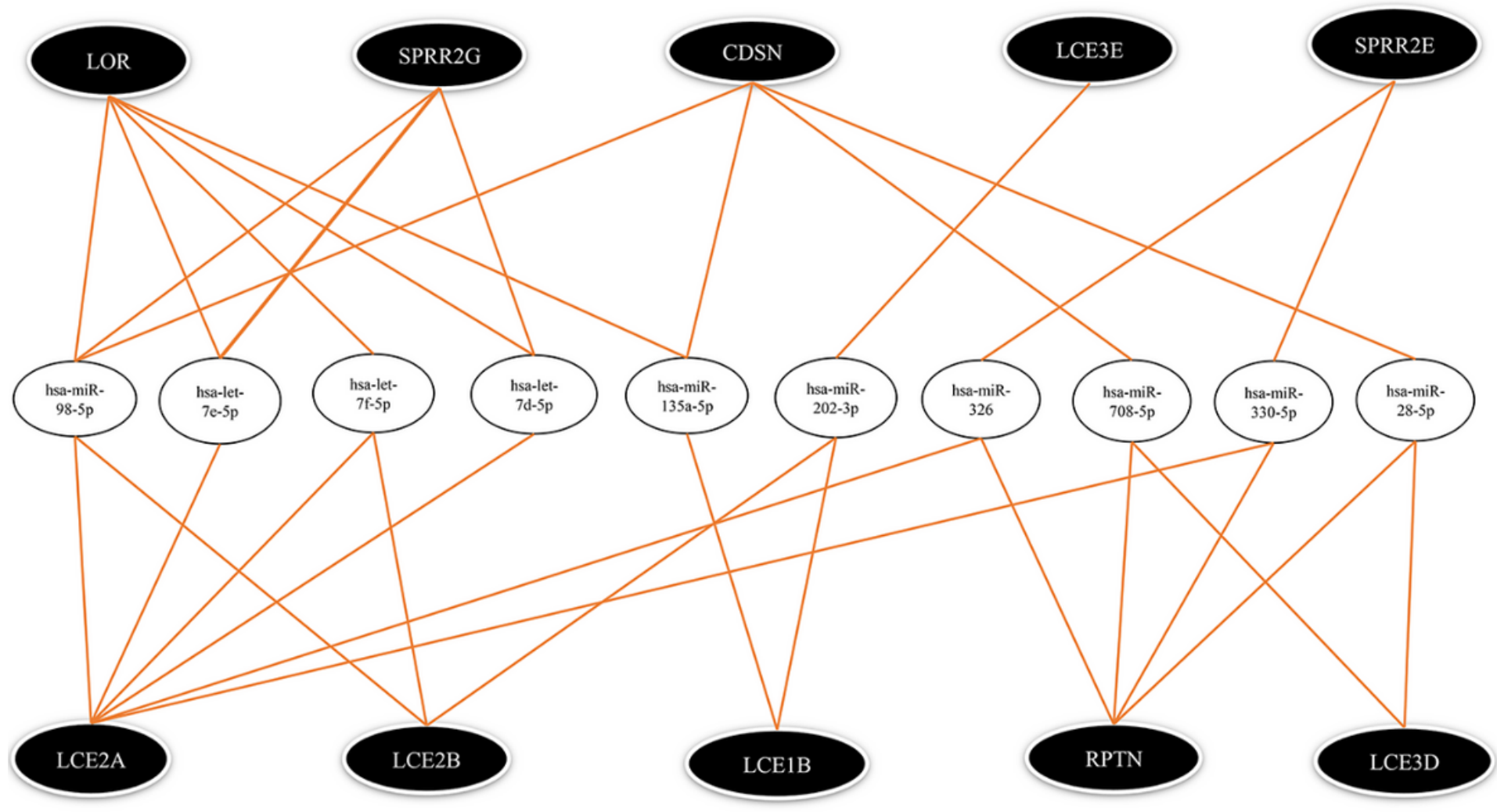

Figure 5 
The collective interaction between the genes and miRNAs.

Page 10/10 